JOURNAL OF THE

AMERICAN MATHEMATICAL SOCIETY

Volume 15, Number 2, Pages 419-442

S 0894-0347(01)00375-7

Article electronically published on December 19, 2001

\title{
STRICHARTZ ESTIMATES \\ FOR SECOND ORDER HYPERBOLIC OPERATORS WITH NONSMOOTH COEFFICIENTS III
}

\author{
DANIEL TATARU
}

\section{INTRODUCTION}

Denote by $x=\left(x_{0}, x_{1}, \ldots, x_{n}\right)=\left(x_{0}, x^{\prime}\right)$ the coordinates in $\mathbb{R} \times \mathbb{R}^{n}$. Sometimes we refer to the first coordinate $x_{0}$ as "time" and to $x^{\prime}$ as "space" coordinates. The Strichartz estimates for solutions to the homogeneous wave equation in $\mathbb{R} \times \mathbb{R}^{n}$,

$$
\square u=0, \quad u(0)=u_{0}, \quad u_{t}(0)=u_{1},
$$

have the form

$$
\left\||D|^{1-\rho} u\right\|_{L^{p}\left(L^{q}\right)} \lesssim\left\|\nabla u_{0}\right\|_{L^{2}}+\left\|u_{1}\right\|_{L^{2}} .
$$

Such an estimate holds for all pairs $(\rho, p, q)$ satisfying the relations $2 \leq p \leq \infty$, $2 \leq q \leq \infty$ and

$$
\frac{1}{p}+\frac{n}{q}=\frac{n}{2}-\rho, \quad \frac{2}{p}+\frac{n-1}{q} \leq \frac{n-1}{2}
$$

with the exception of the forbidden endpoint $(1,2, \infty)$ in dimension $n=3$. All $(\rho, p, q)$ satisfying these relations are called Strichartz pairs in the sequel. If the equality holds in the second part of (1.2), then the corresponding pair is called a sharp Strichartz pair.

A straightforward consequence of (1.1) is an estimate for solutions to the inhomogeneous problem

$$
\square u=f, \quad u(0)=0, \quad u_{t}(0)=0,
$$

namely

$$
\left\||D|^{1-\rho} u\right\|_{L^{p}\left(L^{q}\right)} \lesssim\|f\|_{L^{1}\left(L^{2}\right)} .
$$

The simplest case of (1.3) is the well-known energy estimate

$$
\|\nabla u\|_{L^{\infty}\left(L^{2}\right)} \lesssim\|f\|_{L^{1}\left(L^{2}\right)}
$$

However, there is a larger family of estimates for solutions to the inhomogeneous wave equation where we also vary the norms in the right-hand side,

$$
\left\||D|^{1-\rho} u\right\|_{L^{p}\left(0, T ; L^{q}\right)} \lesssim\left\||D|^{\rho_{1}} f\right\|_{L^{p_{1}^{\prime}\left(L^{q_{1}^{\prime}}\right)}} .
$$

This holds for all Strichartz pairs $(\rho, p, q),\left(\rho_{1}, p_{1}, q_{1}\right)$.

Received by the editors October 12, 1999, and, in revised form, April 12, 2001.

1991 Mathematics Subject Classification. Primary 35L10, 35L70.

Key words and phrases. Strichartz estimates, nonlinear wave equation.

This research was partially supported by NSF grant DMS-9622942 and by an Alfred P. Sloan fellowship. 
Estimates of the above type were first proved in the constant coefficient case in [3], [16]. Further references can be found in a more recent expository article [5]. The endpoint estimate $(p, q)=\left(2, \frac{2(n-1)}{n-3}\right)$ was only recently obtained in $\left.[9] 4\right)$.

In this article we are interested in the variable coefficient case of these estimates, where we replace $\square$ by a second order operator of the form

$$
P(x, D)=\partial_{i} g^{i j}(x) \partial_{j},
$$

which is strongly hyperbolic with respect to time.

If the coefficients $g^{i j}$ are smooth, then the estimates hold locally (see [7, [13]) (except for the endpoint). For $C^{2}$ coefficients, in dimension $n=2,3$, the estimates are proved in [14. On the other hand in [15] they are shown to fail for $C^{s}$ coefficients, $s<2$.

In a previous article [18] we show that the full estimates hold in all dimensions for operators with $C^{2}$ coefficients, and we also obtain appropriate weaker estimates for operators with $C^{s}$ coefficients for $0<s<2$. The main goal of this article is to prove that the estimates are still true if the coefficients have two derivatives in $L^{1}\left(L^{\infty}\right)$, and then to explore some consequences of this result.

In what follows we assume that the matrices $\left(g^{i j}(x)\right),\left(g^{i j}(x)\right)^{-1}$ are uniformly bounded and of signature $(1, n)$. Furthermore, we also assume that the surfaces $x_{0}=$ const are space-like uniformly in $x$, i.e. that $g^{00}>c>0$.

Before we state our results observe that in the variable coefficient case there are at least two parameters that we need to take into account, namely the time $T$ for which the estimates hold and the size of the second derivatives of the coefficients. To these we add, as in [18, a third parameter, $\mu$, related to the constants in the estimates. This third parameter plays an essential role in the applications. Our first result is

Theorem 1.1. Assume that $\nabla^{2} g \in L^{1}\left(L^{\infty}\right)$. Let $(\rho, p, q)$ be a Strichartz pair. Then

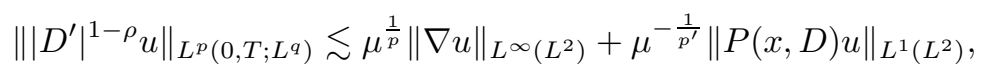

whenever the right-hand side is finite provided that $\mu \geq 1$ and

$$
T\left\|\nabla^{2} g\right\|_{L^{1}\left(L^{\infty}\right)} \leq \mu^{2} .
$$

Observe that the relation (1.7) is nontrivial in terms of scaling since both sides are dimensionless. If we assume in addition that $u$ is compactly supported, then (1.6) holds for all $\mu>0$ since one can use energy estimates to bound the $L^{\infty}\left(L^{2}\right)$ norm of $\nabla u$ by the $L^{1}\left(L^{2}\right)$ norm of $P(x, D) u$. Then it is more convenient to state the estimate in the form

$$
\left\||D|^{-\rho} \nabla u\right\|_{L^{p}\left(0, T ; L^{q}\right)} \leq \mu^{\frac{1}{p}}\|\nabla u\|_{L^{\infty}\left(L^{2}\right)}+\mu^{-\frac{1}{p^{p}}}\|P(x, D) u\|_{L^{1}\left(L^{2}\right)} .
$$

This differs from (1.6) by a microlocal elliptic estimate away from the characteristic set of $P$.

The estimates in Theorem 1.1 also lead to some weaker Strichartz estimates in the case when the coefficients have less regularity. Following the terminology in [19] define the microlocalizable scale of spaces $\mathcal{X}^{s}$ by

$$
\|u\|_{\mathcal{X}^{s}}=\sup _{\lambda} \lambda^{s}\left\|S_{\lambda} u\right\|_{L^{1}\left(L^{\infty}\right)},
$$


where

$$
1=\sum_{\lambda=2^{j}} S_{\lambda}
$$

is a standard Littlewood-Paley decomposition. Then we consider operators with coefficients in the $\mathcal{X}^{s}$ spaces for $0<s<2$. To measure the regularity of the solutions we introduce the homogeneous Besov type spaces $\dot{B}_{s}^{p q r}$ with norms

$$
\|u\|_{\dot{B}_{s}^{p q r}}^{r}=\sum_{\lambda=2^{j}} \lambda^{r s}\left\|S_{\lambda} u\right\|_{L^{p}\left(L^{q}\right)}^{r} .
$$

Theorem 1.2. Assume that $P$ is in divergence form and that $g \in \mathcal{X}^{s}, 0 \leq s<2$. Let $(\rho, p, q)$ be a Strichartz pair and

$$
\sigma=\frac{2-s}{2+s}
$$

Then

$$
\left\||D|^{1-\rho-\frac{\sigma}{p}} u\right\|_{\dot{B}_{0}^{p q \infty}} \lesssim \mu^{\frac{1}{p}}\|\nabla u\|_{L^{\infty}\left(L^{2}\right)}+\mu^{-\frac{1}{p^{\prime}}}\left\||D|^{-\sigma} P u\right\|_{L^{1}\left(L^{2}\right)}
$$

for all u compactly supported in $[0, T]$ and $\mu, T$ satisfying

$$
T^{s}\|g\|_{\mathcal{X}^{s}}^{2} \leq \mu^{2+s} \text {. }
$$

Compared to the full estimates, here we lose $\frac{\sigma}{p}$ derivatives, but, on the other hand, we assume less about the right-hand side of the equation. The reason we use Besov spaces on the left is that in general we do not have square summability of the dyadic pieces on the right. The argument we use for $s=2$ to avoid this problem can be extended to $s>\frac{3}{2}$. Another borderline case is when $\nabla g \in L^{2}\left(L^{\infty}\right)$. Here we do not explore such issues in detail as they are not the main topic of this article. Our main results are the dyadic estimates.

As in Theorem 1.1 if $\mu \geq 1$, then the assumption that $u$ has compact support is no longer needed and, modulo an elliptic estimate, (1.9) is equivalent to

$$
\left\|\left|D^{\prime}\right|^{-\rho-\frac{\sigma}{p}} \nabla u\right\|_{L^{p}\left(\dot{B}_{0}^{q \infty}\right)} \lesssim \mu^{\frac{1}{p}}\|\nabla u\|_{L^{\infty}\left(L^{2}\right)}+\mu^{-\frac{1}{p^{\prime}}}\left\|\left|D^{\prime}\right|^{-\sigma} P u\right\|_{L^{1}\left(L^{2}\right)} .
$$

The estimates for solutions to the homogeneous equation follow easily from the above theorems combined with the energy estimates. Uniform energy estimates for a time $T$ hold for instance if $1 \leq s \leq 2$ and

$$
\left\|\partial_{t} g\right\|_{L^{1}\left(L^{\infty}\right)} \leq 1
$$

Now let us turn our attention to the estimates for the inhomogeneous problem. Our first result is a generalization of (1.6).

Theorem 1.3. Assume that the coefficients satisfy $\nabla^{2} g \in L^{1}\left(L^{\infty}\right)$. Let $(\rho, p, q)$ be a Strichartz pair. Then

$$
\left\|\left|D^{\prime}\right|^{1-\rho} u\right\|_{L^{p}\left(L^{q}\right)} \lesssim \mu^{\frac{1}{p}}\|\nabla u\|_{L^{\infty}\left(L^{2}\right)}+\mu^{-\frac{1}{p^{\prime}}}\left\|f_{1}\right\|_{L^{1}\left(L^{2}\right)}+\left\|\left|D^{\prime}\right|^{\rho} f_{2}\right\|_{L^{p^{\prime}}\left(L^{q^{\prime}}\right)}
$$

for all u satisfying

$$
P(x, D) u=f_{1}+f_{2}
$$

and $\mu \geq 1$ with

$$
T\left\|\nabla^{2} g\right\|_{L^{1}\left(L^{\infty}\right)} \leq \mu^{2} .
$$


The proof of this result is similar to the proof of the corresponding result in [18]. The estimate (1.11) can also be rephrased in a manner similar to (1.8). The analogue result in the case when the coefficients are in $\mathcal{X}^{s}$, with $0 \leq s<2$, is

Theorem 1.4. Assume that $P$ is in divergence form and that $g \in \mathcal{X}^{s}, 0 \leq s<2$. Let $(\rho, p, q)$ be a Strichartz pair. Then

$$
\begin{aligned}
\left\||D|^{1-\rho-\frac{\sigma}{p}} u\right\|_{\dot{B}_{0}^{p q \infty}} & \lesssim \mu^{\frac{1}{p}}\|\nabla u\|_{L^{\infty}\left(L^{2}\right)}+\mu^{-\frac{1}{p^{\prime}}}\left\||D|^{-\sigma} f_{1}\right\|_{L^{1}\left(L^{2}\right)} \\
& +\left\||D|^{\rho-\frac{\sigma}{p}} f_{2}\right\|_{B_{0}^{p^{\prime} q^{\prime} \infty}}
\end{aligned}
$$

for all $u$ compactly supported in $[0, T]$ satisfying $P(x, D) u=f_{1}+f_{2}$ and

$$
T^{s}\|g\|_{\mathcal{X}^{s}}^{2} \leq \mu^{2+s} \text {. }
$$

Applied to solutions to the initial value problem

$$
P(x, D) u=f, \quad u(0)=u_{0}, \quad u_{t}(0)=u_{1}
$$

this shows that the full Strichartz estimates hold for operators whose coefficients have two derivatives in $L^{1}\left(L^{\infty}\right)$.

Corollary 1.5. Assume that $\nabla^{2} g \in L^{1}\left(0,1 ; L^{\infty}\right)$. Let $(\rho, p, q)$ and $\left(\rho_{1}, p_{1}, q_{1}\right)$ be Strichartz pairs. Then the solution $u$ to (1.13) satisfies

$$
\left\|\left|D^{\prime}\right|^{1-\rho_{1}} u\right\|_{L^{p_{1}}\left(0,1 ; L^{q_{1}}\right)} \lesssim\left\|\left|D^{\prime}\right|^{\rho} f\right\|_{L^{p^{\prime}}\left(0,1 ; L^{q^{\prime}}\right)}+\left\|u_{0}\right\|_{H^{1}}+\left\|u_{1}\right\|_{L^{2}}
$$

The corresponding result for $1 \leq s<2$ is

Corollary 1.6. Assume that the operator $P$ has $\mathcal{X}^{s}$ coefficient 1 with $1 \leq s \leq 2$. Let $(\rho, p, q),\left(\rho_{1}, p_{1}, q_{1}\right)$ be Strichartz pairs. Then the solution $u$ to (1.13) satisfies

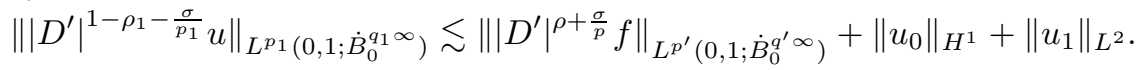

The weaker form of the special case of this result which we use in the study of the nonlinear wave equation corresponds to $s=1$.

Corollary 1.7. Assume that the coefficients of $P$ satisfy $\nabla g \in L^{1}\left(L^{\infty}\right)$. Let $(\rho, p, q)$ be a Strichartz pair. Then the solution $u$ to (1.13) satisfies

$$
\left\|\left|D^{\prime}\right|^{1-\rho_{1}-\frac{1}{3 p}} u\right\|_{L^{p}\left(0,1 ; \dot{B}_{0}^{q \infty}\right)} \lesssim\|f\|_{L^{1}\left(L^{\infty}\right)}+\left\|u_{0}\right\|_{H^{1}}+\left\|u_{1}\right\|_{L^{2}}
$$

\section{The FBI TRANSFORM AND ERROR ESTIMATES}

The essential tool in our proof of the Strichartz estimates is the FBI transform. The technique we use was first developed in [17]. The idea is quite simple, namely to obtain estimates for the FBI transform of the solution. This requires conjugating the operator $P$ with respect to the FBI transform. The first part of [17] is devoted to proving $L^{2}$ error estimates for this conjugation. Here we cannot use directly the error estimates in [17, but the new estimates we prove are similar in spirit.

The FBI transform of a temperate distribution $f$ is a holomorphic function in $\mathbb{C}^{n}$ defined as

$$
\left(T_{\lambda} f\right)(z)=\lambda^{\frac{3 n}{4}} 2^{-\frac{n}{2}} \pi^{-\frac{3 n}{4}} \int e^{-\frac{\lambda}{2}(z-y)^{2}} f(y) d y
$$

\footnotetext{
${ }^{1}$ For $s=1$ this has to be replaced by $\nabla g \in L^{1}\left(L^{\infty}\right)$ so that we still have the energy estimates.
} 
Set

$$
z=x-i \xi, \quad \Phi(z)=e^{-\lambda \xi^{2}} .
$$

Then the operator $T_{\lambda}$ is an isometry from $L^{2}\left(\mathbb{R}^{n}\right)$ onto the closed subspace of holomorphic functions in $L_{\Phi}^{2}\left(\mathbb{C}^{n}\right)$. One inversion formula is provided by the adjoint operator:

$$
f(y)=\lambda^{\frac{3 n}{4}} 2^{-\frac{n}{2}} \pi^{-\frac{3 n}{4}} \int \Phi(z) e^{-\frac{\lambda}{2}(\bar{z}-y)^{2}}\left(T_{\lambda} f\right)(z) d x d \xi .
$$

The FBI transform of a function $f$ can also be expressed in terms of the Fourier transform of $f$,

$$
T_{\lambda} f(z)=\lambda^{\frac{5 n}{4}} 2^{-\frac{n}{2}} \pi^{-\frac{3 n}{4}} e^{\frac{\lambda}{2} \xi^{2}} \int e^{-\frac{\lambda}{2}(\eta-\xi)^{2}} e^{i \lambda x \eta} \hat{f}(\lambda \eta) d \eta .
$$

For additional information on the FBI transform we refer the reader to Delort [4].

Given a compactly supported symbol $a(x, \xi)$ we define the rescaled operators

$$
A_{\lambda}=A\left(x, \frac{D}{\lambda}\right) \text {. }
$$

Then our main result in [17] deals with the problem of conjugating the operator $A_{\lambda}$ with respect to $T_{\lambda}$. All we need here is the result for $C^{2}$ symbols. In this case we set

$$
\tilde{a}_{\lambda}=a+\frac{1}{-i \lambda} a_{x}\left(\partial_{\xi}-\lambda \xi\right)+\frac{1}{\lambda} a_{\xi}\left(\frac{1}{i} \partial_{x}-\lambda \xi\right) .
$$

Since we only consider this operator on holomorphic functions, we can also rewrite it in a complex fashion as

$$
\tilde{a}_{\lambda}=a+\frac{2}{\lambda}(\bar{\partial} a)(\partial-i \lambda \xi), \quad 1<s \leq 2 .
$$

Define the remainder

$$
R_{\lambda, a}=T_{\lambda} A_{\lambda}-\tilde{a}_{\lambda} T_{\lambda}
$$

Then the result in [17] is (see Theorem 1 and Remark 1.2 there):

Theorem 2.1. Assume that $a \in C_{x}^{2}\left(C_{0}^{\infty}\right)$. Then

$$
\left\|R_{\lambda, a}\right\|_{L^{2} \rightarrow L_{\phi}^{2}} \lesssim \lambda^{-1}
$$

and

$$
\left\|\left(\partial_{\xi}-\lambda \xi\right) R_{\lambda, a}\right\|_{L^{2} \rightarrow L_{\phi}^{2}} \lesssim \lambda^{-\frac{1}{2}}
$$

The motivation for the second inequality is that it allows us to compute the regularity of the traces of the remainder on the characteristic cone

$$
K=\{(x, \xi) ; p(x, \xi)=0\}
$$

by using the trace theorem:

Corollary 2.2. Assume that $a \in C_{x}^{2}\left(C_{0}^{\infty}\right)$. Then

$$
\left\|R_{\lambda, a}\right\|_{L^{2} \rightarrow L_{\phi}^{2}(K)} \lesssim \lambda^{-\frac{3}{4}}
$$


In our case we need a modification of Theorem 2.1

Theorem 2.3. Assume that the symbol a satisfies $\partial_{x}^{2} a \in L^{1}\left(L^{\infty}\right)\left(C_{0}^{\infty}\right)$ and that its Fourier transform in $x$ is supported in $B(0, \sqrt{\lambda})$. Then

$$
\begin{gathered}
\left\|\Phi^{\frac{1}{2}} R_{\lambda, a}\right\|_{L^{\infty}\left(L^{2}\right) \rightarrow L^{2}} \lesssim \lambda^{-\frac{3}{4}}, \\
\left\|\Phi^{\frac{1}{2}} R_{\lambda, a}\right\|_{L^{\infty}\left(L^{2}\right) \rightarrow L_{x_{0}}^{1} L_{x^{\prime} \xi^{\prime}}^{2}(K)} \lesssim \lambda^{-\frac{3}{4}} .
\end{gathered}
$$

One can think of this result in terms of the previous one. More precisely, Theorem [2.1] implies Theorem[2.3] when the argument is supported in a time interval of length $\lambda^{-\frac{1}{2}}$. Then the summability with respect to such time intervals comes from the corresponding summability for the second derivatives of $a$.

Proof. Without any restriction in generality we can assume that the symbol $a$ has the form

$$
a(x, \xi)=b(x) c(\xi)
$$

with $\partial_{x}^{2} b \in L^{1}\left(L^{\infty}\right)$ and $c \in C_{0}^{\infty}$. This reduction can be achieved by writing a Fourier series for $a$ as a function of $\xi$ with respect to a larger region and then by truncating the terms in the expansion near the support of $a$.

First we prove the result for $b$, then we use Theorem 2.1 for $c$ to obtain the desired estimate for $b(x) c(\xi)$.

The remainder $\Phi^{\frac{1}{2}} R_{\lambda, b}$ is given by

$$
\Phi^{\frac{1}{2}} R_{\lambda, b} u(x, \xi)=\lambda^{\frac{3(n+1)}{4}} \int e^{i \lambda \xi(x-y)} e^{-\frac{\lambda}{2}(x-y)^{2}}\left(b(x)+b_{x}(x)(y-x)-b(y)\right) u(y) d y .
$$

Using the $T T^{*}$ argument the estimate (2.4) reduces to

$$
\left\|R_{\lambda, b}^{*} \Phi R_{\lambda, b}\right\|_{L^{\infty}\left(L^{2}\right) \rightarrow L^{1}\left(L^{2}\right)} \lesssim \lambda^{-\frac{3}{2}} .
$$

But a simple computation shows that $R_{\lambda, b}^{*} \Phi R_{\lambda, b}$ is a multiplication operator by

$$
f(y)=\lambda^{\frac{n+1}{2}} \int e^{-\lambda(x-y)^{2}}\left(b(x)+b_{x}(x)(y-x)-b(y)\right)^{2} d x .
$$

Then to conclude we need to show that

$$
\|f\|_{L^{1}\left(L^{\infty}\right)} \lesssim \lambda^{-\frac{3}{2}}
$$

This can be easily done using the Taylor series expansion for $b$ together with the Sobolev type estimate

$$
\left\|\partial_{x}^{2} b\right\|_{L^{2}\left(L^{\infty}\right)} \lesssim \lambda^{\frac{1}{4}}\left\|\partial_{x}^{2} b\right\|_{L^{1}\left(L^{\infty}\right)}
$$

which is true when the Fourier transform of $b$ is supported in $|\xi| \leq \lambda^{\frac{1}{2}}$.

To prove the second estimate look first at the restriction of the operator $\Phi^{\frac{1}{2}} R_{\lambda, b}$ acting from a fixed time slice into a fixed time slice. This corresponds to the decomposition

$$
\Phi^{\frac{1}{2}} R_{\lambda, b} u\left(x_{0}\right)_{\mid K}=\lambda^{\frac{3}{4}} \int e^{i \lambda \xi_{0}\left(x_{0}-y_{0}\right)} R_{x_{0}, y_{0}} u\left(y_{0}\right) d y_{0},
$$

where

$$
R_{x_{0}, y_{0}} u\left(x^{\prime}, \xi^{\prime}\right)=\lambda^{\frac{3 n}{4}} \int e^{i \lambda \xi^{\prime}\left(x^{\prime}-y^{\prime}\right)} e^{-\frac{\lambda}{2}(x-y)^{2}}\left(b(x)+b_{x}(x)(y-x)-b(y)\right) u\left(y^{\prime}\right) d y^{\prime} .
$$


We first estimate the operator $R_{x_{0}, y_{0}}$ in $L^{2}$.

Lemma 2.4. Suppose that $\partial_{x}^{2} b \in L^{1}\left(L^{\infty}\right)$. Then

$$
\left\|R_{x_{0}, y_{0}}\right\|_{L^{2} \rightarrow L^{2}} \lesssim \lambda^{-1} e^{-c \lambda\left(x_{0}-y_{0}\right)^{2}} \int_{x_{0}}^{y_{0}}\left\|\left(\partial_{x}^{2} b\right)(s)\right\|_{L^{\infty}} d s .
$$

Proof. The operator $R_{x_{0}, y_{0}}^{*} R_{x_{0}, y_{0}}$ is a multiplication operator by

$$
f\left(y^{\prime}\right)=\lambda^{\frac{n}{2}} \int e^{-\lambda(x-y)^{2}}\left(b(x)+b_{x}(x)(y-x)-b(y)\right)^{2} d x^{\prime} .
$$

Since

$$
\left(b(x)+b_{x}(x)(y-x)-b(y)\right)=\int_{0}^{1} t\left(\partial_{x}^{2} b\right)(t x+(1-t) y)(y-x, y-x) d t,
$$

we obtain

$$
\left|b(x)+b_{x}(x)(y-x)-b(y)\right| \lesssim|x-y|^{2} \int_{x_{0}}^{y_{0}}\left\|\left(\partial_{x}^{2} b\right)(s)\right\|_{L^{\infty}} d s .
$$

Then

$$
\begin{aligned}
\left|f\left(y^{\prime}\right)\right| & \lesssim \lambda^{\frac{n}{2}}\left(\int_{x_{0}}^{y_{0}}\left\|\left(\partial_{x}^{2} b\right)(s)\right\|_{L^{\infty}} d s\right)^{2} \int|x-y|^{4} e^{-\lambda(x-y)^{2}} d x^{\prime} \\
& \lesssim \lambda^{-2} e^{-c\left(x_{0}-y_{0}\right)^{2}}\left(\int_{x_{0}}^{y_{0}}\left\|\left(\partial_{x}^{2} b\right)(s)\right\|_{L^{\infty}} d s\right)^{2} .
\end{aligned}
$$

This implies the estimate in the lemma.

Now we use (2.6) to conclude the proof of Theorem 2.3 for $b$. We have

$$
\begin{aligned}
\left\|\Phi^{\frac{1}{2}} R_{\lambda, b} u_{\mid K}\right\|_{L_{x_{0}}^{1}\left(L_{x^{\prime}, \xi^{\prime}}^{2}\right)} & \lesssim \lambda^{\frac{3}{4}} \int\left\|R_{x_{0} y_{0}}\right\|_{L^{2} \rightarrow L^{2}}\left\|u\left(y_{0}\right)\right\|_{L^{2}} d x_{0} d y_{0} \\
& \lesssim\|u\|_{L^{\infty}\left(L^{2}\right)} \lambda^{\frac{3}{4}} \int\left\|R_{x_{0} y_{0}}\right\|_{L^{2} \rightarrow L^{2}} d x_{0} d y_{0} .
\end{aligned}
$$

Then (2.5) for $b$ follows if we verify that

$$
\int\left\|R_{x_{0} y_{0}}\right\|_{L^{2} \rightarrow L^{2}} d x_{0} d y_{0} \lesssim c \lambda^{-\frac{3}{2}}
$$

But by (2.6) we get

$$
\int\left\|R_{x_{0} y_{0}}\right\|_{L^{2} \rightarrow L^{2}} \lesssim \lambda^{-1} \int \frac{1}{\left|x_{0}-y_{0}\right|} e^{-c \lambda\left(x_{0}-y_{0}\right)^{2}} \int_{x_{0}}^{y_{0}}\left\|\left(\partial_{x}^{2} b\right)(s)\right\|_{L^{\infty}} d s d x_{0} d y_{0} .
$$

We change the order of integration and use scaling to integrate with respect to $x_{0}, y_{0}$. This yields

$$
\int\left\|R_{x_{0} y_{0}}\right\|_{L^{2} \rightarrow L^{2}} \lesssim \lambda^{-\frac{3}{2}} \int\left\|\left(\partial_{x}^{2} b\right)(s)\right\|_{L^{\infty}} d s
$$

which gives 2.7).

Finally, we need to put together the error estimates for $b$ and $c$. We have

$$
R_{\lambda, a}=R_{\lambda, b} C\left(\frac{D}{\lambda}\right)+\left(b+\lambda^{-1}\left(\partial_{x} b\right)\left(\partial_{\xi}-\lambda \xi\right)\right) R_{\lambda, c}
$$


The bounds for the first right-hand side term follow immediately from the bounds for $R_{\lambda, b}$. For the second right-hand side term observe that $b, \partial_{x} b$ are bounded and therefore it suffices to prove the same bounds for the operators

$$
\left.R_{\lambda, c}, \quad \lambda^{-1}\left(\partial_{\xi}-\lambda \xi\right)\right) R_{\lambda, c} .
$$

In both cases these follow from the $L^{2}$ bounds in Theorem 2.1 since $x, \xi$ are in a compact set.

\section{Proof of Theorem 1.1}

The first part of the proof involves several localization type arguments. First we reduce the estimate to the case when $\mu=1$ and $u$ is supported in a cube of size 1 . Then we use a Littlewood-Paley decomposition to reduce the problem to the corresponding dyadic estimates at fixed frequency $\lambda$. Finally we truncate the coefficients of $P$ at frequency $\sqrt{\lambda}$.

A second stage of the proof is to obtain good $L^{2}$ estimates for the FBI transform of $u$. Conjugating the operator $P$ with respect to the FBI transform $T_{\lambda}$ we get two ode's in the "FBI" space. One of these ode's is along the gradient flow of $p(x, \xi)$ and provides an elliptic estimate away from the characteristic cone. The other one is along the Hamilton flow of $p$ and corresponds to propagation of singularities. By exploiting the $L^{2}$ information coming from the two ode's we can reduce the dyadic inequalities to certain oscillatory integral estimates.

The last step is to prove the oscillatory integral estimates, using a complex interpolation argument. This requires sharp estimates on the regularity of the Hamilton flow. This argument is almost identical to the one in [18] and we do not repeat it here. Instead we just study the regularity of the Hamilton flow and set up the oscillatory integrals as in [18.

Reduction to the case $\mu=1$. Because of the scale invariance we can assume without any restriction in generality that $\left\|\partial_{x}^{2} g\right\|_{L^{1}\left(L^{\infty}\right)} \leq 1$ and that $T=\mu^{2}$. The interesting case is when $\rho<1$ since all other estimates follow by Sobolev embeddings.

To achieve the reduction of (1.6) to the case $\mu=1$ decompose the interval $[0, T]$ into maximal subintervals

$$
0=t_{0}<t_{1}<\cdots<t_{k}=T
$$

so that

$$
\|P(x, D) u\|_{L^{1}\left(t_{j}, t_{j+1} ; L^{2}\right)} \leq \mu^{-1}\|P(x, D) u\|_{L^{1}\left(0, T ; L^{2}\right)}
$$

and

$$
\left(t_{j+1}-t_{j}\right)\left\|\partial_{x}^{2} g\right\|_{L^{1}\left(t_{j}, t_{j+1} ; L^{\infty}\right)} \leq 1
$$

for all $j$. We claim that the number $k$ of intervals satisfies

$$
\mu<k<2 \mu \text {. }
$$

The bound from below follows directly from (3.1). For the bound from above observe that the equality must hold either in (3.1) or in (3.2). The number $k_{1}$ of intervals for which the equality holds in (3.1) is at most $\mu$. On an interval in which equality holds in the (3.2), on the other hand, we get

$$
T^{-\frac{1}{2}}\left(t_{j+1}-t_{j}\right)+T^{\frac{1}{2}}\left\|\partial_{x}^{2} g\right\|_{L^{1}\left(t_{j}, t_{j+1} ; L^{\infty}\right)} \geq 2 .
$$


Summing up over all intervals with this property we get

$$
2 T^{\frac{1}{2}} \geq 2 k_{2}
$$

where $k_{2}$ is the number of such intervals. This gives

$$
k_{2} \leq T^{\frac{1}{2}}=\mu
$$

therefore

$$
k \leq k_{1}+k_{2}=2 \mu \text {. }
$$

If we apply the estimate (1.6) for $\mu=1$ on each such interval we obtain

$$
\left\|\left|D^{\prime}\right|^{-\rho} \nabla u\right\|_{L^{p}\left(t_{j}, t_{j+1} ; L^{q}\right)} \lesssim\|\nabla u\|_{L^{\infty}\left(0, T ; L^{2}\right)}+\mu^{-1}\|P(x, D) u\|_{L^{1}\left(0, T ; L^{2}\right)} .
$$

Raise this to the power $p$ and sum up over $j$ to obtain

$$
\left\|\left|D^{\prime}\right|^{-\rho} \nabla u\right\|_{L^{p}\left(0, T ; L^{q}\right)}^{p} \lesssim \mu\|\nabla u\|_{L^{\infty}\left(0, T ; L^{2}\right)}^{p}+\mu^{1-p}\|P(x, D) u\|_{L^{1}\left(0, T ; L^{2}\right)}^{p} .
$$

This implies (1.6) for $u$ in $[0, T]$.

Reduction to a cube of size 1 . If $\mu=1$, then by rescaling we can take $T=1$, $\left\|\partial_{x}^{2} g\right\|_{L^{1}\left(L^{\infty}\right)} \leq 1$. First we observe that it suffices to prove the estimate with homogeneous spaces replaced by inhomogeneous spaces,

$$
\left\|\left\langle D^{\prime}\right\rangle^{-\rho} \nabla u\right\|_{L^{p}\left(0,1 ; L^{q}\right)} \lesssim\|\nabla u\|_{L^{\infty}\left(L^{2}\right)}+\|u\|_{L^{\infty}\left(L^{2}\right)}+\|P(x, D) u\|_{L^{1}\left(L^{2}\right)},
$$

where $\left\langle D^{\prime}\right\rangle=\left(1+\left|D^{\prime}\right|^{2}\right)^{\frac{1}{2}}$. Indeed, in order to obtain (1.6) from (3.3) we can use a Sobolev estimate

$$
\left\|\left|D^{\prime}\right|^{-\rho} S_{0}^{\prime} \nabla u\right\|_{L^{p}\left(L^{q}\right)} \lesssim\left\|S_{0}^{\prime} \nabla u\right\|_{L^{\infty}\left(L^{2}\right)}
$$

for low frequencies and (3.3) for $\left(1-S_{0}^{\prime}\right) u$.

A second observation is that, due to the variation of parameters formula, it suffices to prove the estimate for $H^{1}$ solutions to the homogeneous equation. These we can extend, still in $H^{1}$, to a larger time interval. Then truncating in time we reduce the problem to the case when $u$ is supported in a fixed time interval, say of length 2 , and $P(x, D) u \in L^{2}$.

Since the $H^{1}$ norm of $u$ can be obtained from the energy estimate

$$
\|\nabla u\|_{L^{\infty}\left(0,2 ; L^{2}\right)} \lesssim\|P(x, D) u\|_{L^{2}}
$$

(3.3) reduces to

$$
\left\|\left|D^{\prime}\right|^{-\rho} \nabla u\right\|_{L^{p}\left(0,2 ; L^{q}\right)} \lesssim\|P(x, D) u\|_{L^{2}} .
$$

Now we decompose $P(x, D) u$ in components supported in cubes of size 1 . Due to the the finite speed of propagation this yields a decomposition of $u$ in components supported in cubes of fixed size $R>1$. If the estimate holds in each such cube, then we can sum up and obtain the global estimate since $p, q \geq 2$.

An elliptic estimate. We still need to prove (3.4) for $u$ supported in a cube of size 1 . We claim that it suffices to prove the weaker estimate

$$
\left\||D|^{1-\rho} u\right\|_{L^{p}\left(L^{q}\right)} \lesssim\|P(x, D) u\|_{L^{2}} .
$$

Clearly 3.5 implies (3.4) microlocally away from a conic neighborhood of $\xi^{\prime}=0$. But near $\xi^{\prime}=0$ the operator $P$ is elliptic. Therefore, from the elliptic theory ${ }^{2}$ we obtain that $u$ is $H^{2}$ near $\xi^{\prime}=0$. This is more than sufficient for our estimates 3

\footnotetext{
${ }^{2}$ Note that $P$ has $C^{1}$ coefficients.

${ }^{3} H^{\frac{3}{2}}$ would suffice.
} 
Reduction to dyadic estimates. Let $s(\xi)$ be a smooth symbol supported in $\frac{1}{2} \leq|\xi| \leq 2$ so that

$$
\sum_{j} s\left(2^{-j} \xi\right)=1
$$

Given $\lambda=2^{j} \geq 1$ let

$$
S_{\lambda}=S\left(\frac{D}{\lambda}\right)
$$

be the standard multiplier which truncates at frequency $\lambda$, and let

$$
S_{0}=\sum_{j<0} s\left(2^{-j} \xi\right)
$$

be the multiplier which cuts off the frequencies less than 1 .

We replace (3.5) with the stronger estimate

$$
\left\||D|^{1-\rho} u\right\|_{L^{p}\left(L^{q}\right)} \lesssim\|u\|_{\dot{H}^{1}}+\|P(x, D)\|_{L^{r}\left(L^{2}\right)}
$$

for some $1<r<2$. The motivation for this choice of $r$ is that for $r=1$ it is more difficult to use the Littlewood-Paley theory while for $r=2$ the commutator estimates become harder due to the low regularity of the coefficients.

We claim that (3.6) reduces to the corresponding dyadic estimates,

$$
\lambda^{-\rho}\left\|S_{\lambda} u\right\|_{L^{p}\left(L^{q}\right)} \lesssim\left\|S_{\lambda} u\right\|_{L^{2}}+\lambda^{-1}\left\|P(x, D) S_{\lambda} u\right\|_{L^{r}\left(L^{2}\right)} .
$$

To obtain (3.6) we square (3.7) and use the Littlewood-Paley theory to sum up the dyadic pieces. Then (3.6) follows provided that we can handle the commutators and show that

$$
\sum_{\lambda=2^{j}}\left\|\left[P, S_{\lambda}\right] u\right\|_{L^{r}\left(L^{2}\right)}^{2} \lesssim\|u\|_{H^{1}}^{2}
$$

or equivalently

$$
\sum_{\lambda=2^{j}}\left\|\left[g, S_{\lambda}\right] \nabla v\right\|_{L^{r}\left(L^{2}\right)}^{2} \lesssim\|v\|_{L^{2}}^{2}
$$

for $v$ compactly supported. If $\tilde{S}_{\lambda}$ is another multiplier whose support is slightly larger, then we can rewrite the operator on the left as

$$
\begin{aligned}
{\left[g, S_{\lambda}\right] \nabla } & =S_{\lambda}(\nabla g)+\left[g, \nabla S_{\lambda}\right] \\
& =S_{\lambda}(\nabla g)+\left[g, \nabla S_{\lambda}\right] \tilde{S}_{\lambda}-\nabla S_{\lambda} g\left(1-\tilde{S}_{\lambda}\right) .
\end{aligned}
$$

The estimate for the first term $S_{\lambda}(\nabla g)$ is trivial. For the second term we get the orthogonality from $\tilde{S}_{\lambda}$ so it suffices to know the commutator estimate

$$
\left[g, \nabla S_{\lambda}\right]: L^{2} \rightarrow L^{2}
$$

This holds since $g$ is Lipschitz (see Taylor [19]). Finally, for the third term it suffices to show that

$$
\left\|\nabla S_{\lambda} g\left(1-\tilde{S}_{\lambda}\right)\right\|_{L^{2} \rightarrow L^{r}\left(L^{2}\right)} \lesssim \lambda^{\frac{r-2}{2 r}} .
$$

Since the supports of the symbols $S_{\lambda}$ and $\left(1-\tilde{S}_{\lambda}\right)$ are $O(\lambda)$ away, it follows that only the frequencies of size $\lambda$ and higher in $g$ are relevant. In other words, if $U_{\lambda}$ is a multiplier selecting the frequencies of size $\lambda$ and higher, then

$$
\nabla S_{\lambda} g\left(1-\tilde{S}_{\lambda}\right)=\nabla S_{\lambda}\left(U_{\frac{\lambda}{10}} g\right)\left(1-\tilde{S}_{\lambda}\right)
$$


therefore

$$
\begin{aligned}
\left\|\nabla S_{\lambda} g\left(1-\tilde{S}_{\lambda}\right)\right\|_{L^{2} \rightarrow L^{r}\left(L^{2}\right)} & \lesssim\left\|U_{\lambda} \nabla g\right\|_{L^{\frac{2 r}{2-r}}\left(L^{\infty}\right)} \\
& \lesssim\left\|U_{\lambda} \nabla g\right\|_{L^{\infty} \frac{2-r}{2 r}}^{1-}\left\|U_{\lambda} \nabla g\right\|_{L^{1}\left(L^{\infty}\right)}^{\frac{2-r}{2 r}} \\
& \lesssim \lambda^{\frac{r-2}{2 r}} .
\end{aligned}
$$

At the last step we have used the fact that $\nabla u \in L^{\infty}$ and $\nabla^{2} u \in L^{1}\left(L^{\infty}\right)$.

This concludes the reduction to the dyadic estimates. As the reader may have noticed, up to this point we have had a large leeway as far as the function spaces are concerned. Naively, the reason for this is that in the FBI calculus we need two derivatives on the coefficients for each derivative in the error estimates, while in the usual commutator estimates one derivative on the coefficients suffices. Now we return to the original set-up and strengthen (3.6) to

$$
\lambda^{-\rho}\left\|S_{\lambda} u\right\|_{L^{p}\left(L^{q}\right)} \lesssim\left\|S_{\lambda} u\right\|_{L^{\infty}\left(L^{2}\right)}+\lambda^{-1}\left\|P S_{\lambda} u\right\|_{L^{1}\left(L^{2}\right)} .
$$

Truncating the coefficients at frequency $\sqrt{\lambda}$. We claim that it suffices to prove the dyadic estimate (3.9) when the coefficients have Fourier transform supported in $|\xi| \leq \sqrt{\lambda}$.

Given $\lambda>0$ define the multiplier

$$
U_{\lambda}=\sum_{j \geq 0} S_{2^{j} \lambda},
$$

which selects the frequencies $\lambda$ and higher. Then we replace $g^{i j}$ by $\left(1-U_{\epsilon \sqrt{\lambda}}\right) g^{i j}$ where $\epsilon$ is a small parameter. Thus we modify the factor $\left\|P S_{\lambda} u\right\|_{L^{1}\left(L^{2}\right)}$ in (3.9) at most by

$$
\left\|\left(U_{\epsilon \sqrt{\lambda}} g^{i j}\right) \partial_{i} \partial_{j} S_{\lambda} u\right\|_{L^{1}\left(L^{2}\right)} \lesssim \lambda\left\|D^{2} g\right\|_{L^{1}\left(L^{\infty}\right)}\|u\|_{L^{\infty}\left(L^{2}\right)} .
$$

But this is controlled by the first right-hand side term. Hence it suffices to prove the estimates with $g^{i j}$ replaced by $\left(1-U_{\epsilon \sqrt{\lambda}}\right) g^{i j}$, which have Fourier transform supported in $|\xi|<\lambda^{\frac{1}{2}}$. In particular, since the coefficients of $p$ are Lipschitz, it follows that

$$
|\Delta p(x, \xi)| \ll \lambda^{\frac{1}{2}}, \quad \frac{1}{4} \leq|\xi| \leq 4 .
$$

Once we have truncated the coefficients we can use again the $L^{2}$ well-posedness of the hyperbolic problem to reduce the estimate to

$$
\lambda^{-\rho}\left\|S_{\lambda} u\right\|_{L^{p}\left(L^{q}\right)} \lesssim\left\|S_{\lambda} u\right\|_{L^{\infty}\left(L^{2}\right)}+\lambda^{-1}\left\|P S_{\lambda} u\right\|_{L^{2}} .
$$

This is a minor reduction which simplifies a bit the analysis of the function $g$ below.

Using the FBI transform. Set

$$
w=\Phi^{\frac{1}{2}} T_{\lambda} S_{\lambda} u .
$$

Then we try to get good $L^{2}$ estimates for $w$. The function $S_{\lambda} u$ can then be recovered from

$$
S_{\lambda} u=T_{\lambda}^{*} \Phi^{\frac{1}{2}} w .
$$

Observe first that $w$ is concentrated in the region

$$
U=\left\{|x| \leq 2, \quad \frac{1}{4} \leq|\xi| \leq 4\right\}
$$


Outside this region we have

$$
\|w\|_{L^{2}\left(U^{c}\right)} \leq e^{-c \lambda}\left\|S_{\lambda} u\right\|_{L^{2}}
$$

which is a straightforward consequence of the representation formula for the FBI transform in terms of the Fourier transform of the argument.

Hence it suffices to get good estimates for $w$ in the region $U$. Set

$$
(\lambda p+2(\bar{\partial} p)(\partial-i \lambda \xi)) \Phi^{-\frac{1}{2}} w=\Phi^{-\frac{1}{2}} g
$$

where

$$
g=\Phi^{\frac{1}{2}}\left(\lambda R_{\lambda, p} S_{\lambda} u+\lambda^{-1} T_{\lambda} P(x, D) S_{\lambda} u\right) .
$$

We can use the error estimates to get good bounds for $g$ in terms of the right-hand side of 3.11.

Lemma 3.1. The following estimates hold:

$$
\begin{gathered}
\|g\|_{L^{2}} \leq \lambda^{\frac{1}{4}}\left\|S_{\lambda} u\right\|_{L^{\infty}\left(L^{2}\right)}+\lambda^{-1}\left\|P(x, D) S_{\lambda} u\right\|_{L^{2}}, \\
\left\|g_{\mid K}\right\|_{L_{x_{0}}^{1}\left(L_{x^{\prime} \xi^{\prime}}^{2}\right)} \lesssim \lambda^{\frac{1}{4}}\left\|S_{\lambda} u\right\|_{L^{\infty}\left(L^{2}\right)}+\lambda^{-1}\left\|P(x, D) S_{\lambda} u\right\|_{L^{2} .}
\end{gathered}
$$

In (3.14) the $L_{\xi^{\prime}}^{2}$ norm of $g_{\mid K}$ is taken with respect to the surface measure on $K$. The information about $g$ in this lemma is all we need in the sequel.

The first order equation (3.12). Since $\Phi^{-\frac{1}{2}} w$ is holomorphic as a function of $z=x-i \xi$, we have

$$
(\partial-i \lambda \xi) \Phi^{-\frac{1}{2}} w=\Phi^{-\frac{1}{2}}\left(\partial_{x}-i \lambda \xi\right) w=i\left(\partial_{\xi}-\lambda \xi\right) \Phi^{-\frac{1}{2}} w=i \Phi^{-\frac{1}{2}} \partial_{\xi} w .
$$

(Recall that $\Phi=e^{-\lambda \xi^{2}}$.) Then from (3.12) we obtain in effect two pieces of information, namely

$$
\left[\left(p_{x} \partial_{\xi}-p_{\xi} \partial_{x}\right)-i \lambda\left(p-p_{\xi} \cdot \xi\right)\right] w=-i g,
$$

respectively

$$
\left[\left(p_{x} \partial_{x}+p_{\xi} \partial_{\xi}\right)+\lambda\left(p-i p_{x} \cdot \xi\right)\right] w=g .
$$

The first equation is an ode along the Hamilton flow of $p$, while the second equation is an ode along the gradient curves of $p$. Our strategy is now to use (3.15) to obtain good estimates for $w$ on the characteristic cone $K$, and then to use (3.16) to obtain good decay rates away from the cone. For all the analysis away from the cone we only use the fact that the coefficients are Lipschitz. The $L^{1}\left(L^{\infty}\right)$ bound on the second derivatives of $g$ is only needed for the analysis of the Hamilton flow on the cone.

If we take the inner product of (3.16) with $p w$ and integrate by parts, then we obtain

$$
\lambda\|p w\|_{L^{2}}^{2}=\frac{1}{2}\left\langle\left(|\nabla p|^{2}+p \Delta p\right) w, w\right\rangle+\Re\langle p w, g\rangle .
$$

Then by (3.10) we get

$$
\lambda^{\frac{1}{2}}\|p w\|_{L^{2}} \lesssim\|w\|_{L^{2}}+\lambda^{-\frac{1}{2}}\|g\|_{L^{2}} .
$$

Away from the characteristic cone this estimate improves the $L^{2}$ bound on $w$ by a $\lambda^{-\frac{1}{2}}$ factor. In the physical space this corresponds to a gain of $1 / 2$ derivative, which suffices for the Strichartz estimates simply by the Sobolev embeddings. Then 
we can cut away this region and assume without any restriction of generality that $w, g$ are supported in a neighborhood of the cone.

We use (3.16) to decompose $w$ into two parts,

$$
w=w_{1}+w_{2},
$$

where $w_{1}$ solves the inhomogeneous equation

$$
\left[\left(p_{x} \partial_{x}+p_{\xi} \partial_{\xi}\right)+\lambda\left(p-i p_{x} \cdot \xi\right)\right] w_{1}=-i g, \quad w_{1 \mid K}=0,
$$

and $w_{2}$ solves the homogeneous equation

$$
\left[\left(p_{x} \partial_{x}+p_{\xi} \partial_{\xi}\right)+\lambda\left(p-i p_{x} \cdot \xi\right)\right] w_{2}=0, \quad w_{2 \mid K}=w .
$$

Similarly we split the corresponding part of $S_{\lambda} u$ into $u_{1}+u_{2}$ with

$$
u_{i}=T_{\lambda}^{*} \Phi^{\frac{1}{2}} a(x, \xi) w_{i},
$$

where $a$ is a smooth cutoff selecting a small neighborhood of $K \cap U$.

The estimate for $w_{1}$. If we multiply (3.18) by $\frac{w_{1}}{p}$ and integrate by parts in a neighborhood $K_{\epsilon}=\{|p| \leq \epsilon\}$ of $K$ we obtain

$$
\begin{aligned}
2 \Re\left\langle\frac{w_{1}}{p}, g\right\rangle_{K_{\epsilon}} & =2 \lambda\left\|w_{1}\right\|_{L^{2}\left(K_{\epsilon}\right)}^{2}+\left\|\frac{|\nabla p|}{p} w_{1}\right\|_{L^{2}\left(K_{\epsilon}\right)}^{2}-\left\langle\frac{\Delta p}{p} w_{1}, w_{1}\right\rangle_{K_{\epsilon}} \\
& +\left\|\frac{|\nabla p|}{p} w_{1}\right\|_{L^{2}(\{|p|=\epsilon\})}^{2} \cdot
\end{aligned}
$$

By (3.10) this implies

$$
\lambda\left\|w_{1}\right\|_{L^{2}\left(K_{\epsilon}\right)}^{2} \lesssim\|g\|_{L^{2}\left(K_{\epsilon}\right)}^{2} .
$$

On the other hand, if we multiply (3.18) by $p w_{1}$ and integrate by parts we obtain the analogue of (3.17) in $K_{\epsilon}$. Summing this with (3.10) we obtain

$$
\lambda^{2}\left\|p w_{1}\right\|_{L^{2}\left(K_{\epsilon}\right)}^{2}+\lambda\left\|w_{1}\right\|_{L^{2}\left(K_{\epsilon}\right)}^{2} \lesssim\|g\|_{L^{2}\left(K_{\epsilon}\right)}^{2} .
$$

Then, if we use the bound (3.13) for $g$, the bound for $u_{1}$ corresponding to (3.9) follows from

$$
\left\|T_{\lambda} \Phi^{\frac{1}{2}} a(x, \xi) w_{1}\right\|_{L^{p}\left(L^{q}\right)} \leq \lambda^{\rho+\frac{1}{4}}\left(\lambda^{\frac{1}{2}}\left\|p w_{1}\right\|^{2}+\left\|w_{1}\right\|^{2}\right),
$$

which is equivalent to

$$
\left\|T_{\lambda}^{*} \Phi^{\frac{1}{2}} \frac{\lambda^{\frac{1}{4}} a(x, \xi)}{1+\lambda^{\frac{1}{2}}|p|}\right\|_{L^{2} \rightarrow L^{p}\left(L^{q}\right)} \leq \lambda^{\rho+\frac{1}{2}} .
$$

By the " $T T^{*}$ " argument this reduces to

$$
\left\|T_{\lambda}^{*} \Phi \frac{\lambda^{\frac{1}{2}} a^{2}(x, \xi)}{\left(1+\lambda^{\frac{1}{2}}|p|\right)^{2}} T_{\lambda}\right\|_{L^{p^{\prime}\left(L^{q^{\prime}}\right) \rightarrow L^{p}\left(L^{q}\right)}} \leq \lambda^{2 \rho+1} .
$$

The weight inside is integrable across the level sets of $p$, therefore we can foliate with respect to the level sets of $p$ and reduce this to

$$
\left\|T_{\lambda}^{*} \Phi a^{2}(x, \xi) \delta_{p=0} T_{\lambda}\right\|_{L^{p^{\prime}}\left(L^{q^{\prime}}\right) \rightarrow L^{p}\left(L^{q}\right)} \leq \lambda^{2 \rho+1},
$$

which was proved in [17]. 
The gradient flow. The next step in our analysis is to estimate $w_{2}$ using (3.19). To achieve this we need to compute the regularity of the gradient flow. Suppose we start with initial data $(x, \xi)$ on the cone $K=\{p=0\}$. Denote by $q$ the natural parameter along the flow, chosen so that $q=0$ on $K$. Set $\left(x_{q}, \xi_{q}\right)$ to be the image of $(x, \xi)$ along the flow. Then $\left(x_{q}, \xi_{q}\right)$ solve the equations

$$
\begin{cases}\partial_{q} x_{q}=p_{x}\left(x_{q}, \xi_{q}\right), & x(0)=x \\ \partial_{q} \xi_{q}=p_{\xi}\left(x_{q}, \xi_{q}\right), & \xi(0)=\xi\end{cases}
$$

Theorem 3.2. Assume that $P$ has $C^{1}$ coefficients with Fourier transform supported in $B(0, \sqrt{\lambda})$. Then

$$
\begin{array}{rlrl}
\left|\partial_{x}^{\alpha} \partial_{\xi}^{\beta} x_{q}\right| & \leq c_{\alpha, \beta} \lambda^{\frac{|\alpha|-1}{2}} e^{c_{\alpha \beta} \sqrt{\lambda}|q|}, & & |\alpha|+|\beta|>0, \\
\left|\partial_{x}^{\alpha} \partial_{\xi}^{\beta}\left(\xi_{q}-\xi\right)\right| \leq c_{\alpha, \beta} \lambda^{\frac{|\alpha|-1}{2}} e^{c_{\alpha \beta} \sqrt{\lambda}|q|}, & & |\alpha|+|\beta|>0 .
\end{array}
$$

Thus the gradient flow blows up exponentially on the $\lambda^{\frac{1}{2}}$ scale. This is, however, compensated by the fact that the fundamental solution to 3.19) exhibits Gaussian decay on precisely the same scale.

Proof. The linearization of the gradient flow is given by the system

$$
\left\{\begin{array}{l}
\partial_{q} y=p_{x x} y+p_{x \xi} \eta \\
\partial_{q} \eta=p_{\xi x} y+p_{\xi \xi} \eta
\end{array}\right.
$$

This implies the inequalities

$$
\left\{\begin{array}{l}
\left|\partial_{q} y\right| \leq c(\sqrt{\lambda}|y|+|\eta|) \\
\left|\partial_{q} \eta\right| \leq c(|y|+|\eta|)
\end{array}\right.
$$

If we take $(y, \eta)=\left(\partial_{x} x_{q}, \partial_{x} \xi_{q}\right)$, then the Cauchy data in (3.22) is $(I, 0)$. Thus we obtain the bounds

$$
\left|\partial_{x} x_{q}\right| \leq e^{c \sqrt{\lambda}|q|}, \quad\left|\partial_{x} \xi_{q}\right| \leq \lambda^{-\frac{1}{2}} e^{c \sqrt{\lambda}|q|} .
$$

If we take $(y, \eta)=\left(\partial_{\xi} x_{q}, \partial_{\xi} \xi_{q}\right)$, then the Cauchy data in (3.22) is $(0, I)$. Then we get the bounds

$$
\left|\partial_{\xi} x_{q}\right| \leq \lambda^{-\frac{1}{2}} e^{c \sqrt{\lambda}|q|}, \quad\left|\partial_{\xi}\left(\xi_{q}-\xi\right)\right| \leq \lambda^{-\frac{1}{2}} e^{c \sqrt{\lambda}|q|} .
$$

The rest follows by induction.

The oscillatory integral for $u_{2}$. Clearly $u_{2}$ can be expressed in terms of the trace of $w$ on the cone. This computation is carried out in the following theorem:

Theorem 3.3. Assume that $P$ has $C^{1}$ coefficients frequency localized in $|\xi| \leq \sqrt{\lambda}$. Then we have

$$
u_{2}=\lambda^{-\frac{1}{2}} V_{\lambda} w_{\mid K}
$$

where $V_{\lambda}$ is an integral operator,

$$
V_{\lambda} w=\lambda^{\frac{3(n+1)}{4}} \int_{K} e^{i \lambda \xi(x-y)} G(x, y, \xi) w d x d \xi
$$

with a kernel $G$ satisfying

$$
\left|\partial_{x}^{\alpha} \partial_{\xi}^{\beta} G(x, y, \xi)\right| \leq c_{\alpha, \beta} \lambda^{\frac{|\alpha|}{2}} e^{-c \lambda(x-y)^{2}} .
$$


Proof. We have

$$
w_{2}(y)=\lambda^{\frac{3(n+1)}{4}} \int e^{i \lambda \xi(y-x)} e^{-\frac{\lambda}{2}(x-y)^{2}} w_{2}(x, \xi) d x d \xi .
$$

Now we choose $q=0$ on the cone and $(x, \xi) \in K$. Then $(q, x, \xi)$ can be interpreted as a new set of coordinates by

$$
(q, x, \xi) \rightarrow\left(x_{q}, \xi_{q}\right)
$$

In these new coordinates we write

$$
w_{2}(y)=\lambda^{\frac{3(n+1)}{4}} \int e^{i \lambda \xi_{q}\left(y-x_{q}\right)} e^{-\frac{\lambda}{2}\left(x_{q}-y\right)^{2}} w_{2}\left(x_{q}, \xi_{q}\right) d x_{q} d \xi_{q} .
$$

Since $w_{2}$ solves the ode (3.19) we can represent it as

$$
w_{2}\left(x_{q}, \xi_{q}\right)=F(x, \xi, q) w(x, \xi),
$$

where $F$ is the solution to the homogeneous equation

$$
\left[\partial_{q}+\lambda\left(p\left(x_{q}, \xi_{q}\right)-i p_{\xi}\left(x_{q}, \xi_{q}\right) \cdot \xi_{q}\right)\right] F=0, \quad F(x, \xi, 0)=1 .
$$

After the change of variable, $w_{2}$ has the form

$$
w_{2}(y)=\lambda^{\frac{3(n+1)}{4}} \int_{R} \int_{K} e^{i \lambda \xi_{q}\left(y-x_{q}\right)} e^{-\frac{\lambda}{2}\left(x_{q}-y\right)^{2}} F(x, \xi, q) \frac{\partial\left(x_{q}, \xi_{q}\right)}{\partial(x, \xi, q)} w(x, \xi) d x d \xi d q
$$

or, further recombining the exponents,

$$
\begin{aligned}
w_{2}(y)=\lambda^{\frac{3(n+1)}{4}} \int_{R} \int_{K} e^{i \lambda \xi(y-x)} e^{i \lambda\left(\xi_{q}-\xi\right)\left(y-x_{q}\right)} e^{-\frac{\lambda}{2}\left(x_{q}-y\right)^{2}} e^{i \lambda \xi\left(x-x_{q}\right)} F(x, \xi, q) \\
\times \frac{\partial\left(x_{q}, \xi_{q}\right)}{\partial(x, \xi, q)} w(x, \xi) d x d \xi d q .
\end{aligned}
$$

Then

$$
G(x, \xi, y)=\lambda^{\frac{1}{2}} \int_{R} e(x, \xi, y, q) d q
$$

where

$$
e(x, \xi, y, q)=e^{i \lambda\left(\xi_{q}-\xi\right)\left(y-x_{q}\right)} e^{-\frac{\lambda}{2}\left(x_{q}-y\right)^{2}} e^{i \lambda \xi\left(x-x_{q}\right)} F(x, \xi, q) \frac{\partial\left(x_{q}, \xi_{q}\right)}{\partial(x, \xi, q)} .
$$

Hence in order to obtain (3.23) it suffices to show that

$$
\left|\partial_{x}^{\alpha} \partial_{\xi}^{\beta} e(x, \xi, y, q)\right| \leq c_{\alpha, \beta} \lambda^{\frac{|\alpha|}{2}} e^{-c \lambda(x-y)^{2}} e^{-c \lambda q^{2}} .
$$

This, in turn, would follow from

$$
\begin{gathered}
\left|\partial_{x}^{\alpha} \partial_{\xi}^{\beta} e^{i \lambda\left(\xi_{q}-\xi\right)\left(y-x_{q}\right)} e^{-\frac{\lambda}{2}\left(x_{q}-y\right)^{2}}\right| \leq c_{\alpha, \beta} \lambda^{\frac{|\alpha|}{2}} e^{-c \lambda\left(x_{q}-y\right)^{2}} e^{c \sqrt{\lambda} q}, \\
\left|\partial_{x}^{\alpha} \partial_{\xi}^{\beta} \frac{\partial\left(x_{q}, \xi_{q}\right)}{\partial(x, \xi, q)}\right| \leq c_{\alpha, \beta} \lambda^{\frac{|\alpha|}{2}} e^{c \sqrt{\lambda} q}, \\
\left|\partial_{x}^{\alpha} \partial_{\xi}^{\beta} e^{i \lambda \xi\left(x-x_{q}\right)} F(x, \xi, q)\right| \leq c_{\alpha, \beta} \lambda^{\frac{|\alpha|}{2}} e^{-c \lambda q^{2}} .
\end{gathered}
$$

The bounds (3.24) and (3.25) follow easily from Theorem 3.2, For 3.26) observe that

$$
F(x, \xi, q)=e^{\lambda \int_{0}^{q} i p_{x}\left(x_{s}, \xi_{s}\right) \xi_{s}-p\left(x_{s}, \xi_{s}\right) d s},
$$


therefore

$$
e^{i \lambda \xi\left(x-x_{q}\right)} F(x, \xi, q)=e^{\lambda \int_{0}^{q} i p_{x}\left(x_{s}, \xi_{s}\right)\left(\xi_{s}-\xi\right)-p\left(x_{s}, \xi_{s}\right) d s} .
$$

Since $p \approx c q$ we obtain the desired Gaussian decay, therefore it remains to show that

$$
\lambda\left|\partial_{x}^{\alpha} d_{\xi}^{\beta}\left(i p_{x}\left(x_{q}, \xi_{q}\right)\left(\xi_{q}-\xi\right)-p\left(x_{q}, \xi_{q}\right)\right)\right| \leq c_{\alpha, \beta} \lambda^{\frac{|\alpha|+1}{2}} e^{c \sqrt{\lambda} q} .
$$

This again follows from Theorem 3.2 .

Taking into account the bound (3.14), we need to prove the estimate

$$
\left\|V_{\lambda} w\right\|_{L^{p}\left(L^{q}\right)} \leq \lambda^{\rho+\frac{1}{4}}\left\|H_{p} w\right\|_{L_{x_{0}}^{1}\left(L_{x^{\prime} \xi^{\prime}}^{2}\right)(K)}
$$

for all $w$ supported in $K \cap U$.

Given a pair $(x, \xi)$ we denote by $\left(x_{t}, \xi_{t}\right)$ its image along the Hamilton flow. This map is homogeneous of order 1 with respect to $\xi$. Then, (3.27) is equivalent to

Theorem 3.4. Let $a(x, \xi)$ be a smooth compactly supported function, which is 0 near $\xi=0$ and 1 in $1 / 4 \leq|\xi| \leq 4$. Then

$$
\left\|V_{\lambda} a(x, \xi) L\right\|_{L^{2}\left(K \cap\left\{x_{0}=0\right\}\right) \rightarrow L^{p}\left(L^{q}\right)} \leq \lambda^{\rho+\frac{1}{4}},
$$

where $L$ is the transport operator along the Hamilton flow given by

$$
(L w)(x, \xi)=\left\{\begin{array}{lll}
0 & \text { if } \quad x_{0}<0, \\
w\left(x_{t}, \xi_{t}\right) & \text { if } \quad x_{t 0}=0, \quad x_{0} \geq 0 .
\end{array}\right.
$$

This is further equivalent to the corresponding bound for the operator

$$
Z=\left(V_{\lambda} a L\right)\left(V_{\lambda} a L\right)^{*}=V_{\lambda} a L L^{*} \delta_{p(x, \xi)=0} a V_{\lambda}^{*},
$$

namely

$$
\|Z\|_{L^{p^{\prime}\left(L^{q^{\prime}}\right) \rightarrow L^{p}\left(L^{q}\right)}} \leq \lambda^{2 \rho+\frac{1}{2}} .
$$

The operator $L L^{*}$ is an integral operator along bicharacteristics, with kernel

$$
l(t, s)=1_{\{t \geq 0, s \geq 0\}} .
$$

This embeds into the analytic family

$$
L_{\theta}=(\theta-1)(t-s)^{\theta-1} l(t, s) .
$$

From this point forward, the proof of (3.30) is identical to the proof in [18] provided that we obtain the same regularity results for the Hamilton flow. This is done in the next section.

The regularity of the Hamilton flow. Here we obtain precise bounds on the derivatives of the flow map $H_{t}$ with respect to $\xi$. Observe first that if the coefficients $g^{i j}$ have Fourier transform supported in $|\xi| \leq \sqrt{\lambda}$, then the following relations hold:

$$
\begin{gathered}
\left\|\partial_{x}^{\alpha} g\right\|_{L^{1}\left(L^{\infty}\right)} \leq c_{\alpha} \lambda^{\frac{\alpha-2}{2}}, \quad|\alpha| \geq 2, \\
\left\|\partial_{x}^{\alpha} g\right\|_{L^{\infty}} \leq c_{\alpha} \lambda^{\frac{\alpha-1}{2}}, \quad|\alpha| \geq 1 .
\end{gathered}
$$


Then

Lemma 3.5. Assume that the coefficients of $P$ satisfy $\nabla^{2} g \in L^{1}\left(L^{\infty}\right)$, with Fourier transform supported in $B(0, \sqrt{\lambda})$. Then the following bounds hold:

$$
\begin{aligned}
& \left|\partial_{\xi}^{\alpha} x_{t}\right| \leq t(1+t \sqrt{\lambda})^{|\alpha|-1}, \quad|\alpha| \geq 1 \\
& \left|\partial_{\xi}^{\alpha} \xi_{t}\right| \leq(1+t \sqrt{\lambda})^{|\alpha|-1}, \quad|\alpha| \geq 1 .
\end{aligned}
$$

Proof. We use induction with respect to $\alpha$. For $\alpha=1$ the functions $\left(\partial_{\xi} x_{t}, d_{\xi} \xi_{t}\right)$ solve the linearized system

$$
\frac{d}{d t}\left(\begin{array}{c}
y \\
\eta
\end{array}\right)=A(t)\left(\begin{array}{c}
y \\
\eta
\end{array}\right), \quad A(t)=\left(\begin{array}{cc}
p_{\xi x}\left(x_{t}, \xi_{t}\right) & p_{\xi \xi}\left(x_{t}, \xi_{t}\right) \\
-p_{x x}\left(x_{t}, \xi_{t}\right) & -p_{x \xi}\left(x_{t}, \xi_{t}\right)
\end{array}\right)
$$

with initial data

$$
\left(\begin{array}{c}
y(0) \\
\eta(0)
\end{array}\right)=\left(\begin{array}{c}
0 \\
I_{n}
\end{array}\right)
$$

Since $A(t)$ is integrable, it follows that the solutions remain bounded. Furthermore, the Cauchy data of the first component is 0 , therefore the first component can be bounded by $c t$.

For the induction step, compute

$$
\frac{d}{d t} \partial_{\xi}^{\alpha} x_{t}=\partial_{\xi}^{\alpha} \frac{d}{d t} x_{t}=\partial_{\xi}^{\alpha} p_{\xi}\left(x_{t}, \xi_{t}\right)
$$

and

$$
\frac{d}{d t} \partial_{\xi}^{\alpha} \xi_{t}=\partial_{\xi}^{\alpha} \frac{d}{d t} \xi_{t}=-\partial_{\xi}^{\alpha} p_{x}\left(x_{t}, \xi_{t}\right)
$$

Then the functions $\left(\partial_{\xi}^{\alpha} x_{t}, \partial_{\xi}^{\alpha} \xi_{t}\right)$ solve a system of the form

$$
\frac{d}{d t}\left(\begin{array}{c}
y \\
\eta
\end{array}\right)=A(t)\left(\begin{array}{c}
y \\
\eta
\end{array}\right)+\left(\begin{array}{c}
\bar{y}(t) \\
\bar{\eta}(t)
\end{array}\right)
$$

with zero initial data, where the inhomogeneous term $(\bar{y}(t), \bar{\eta}(t))$ can be expressed in terms of the lower order derivatives as a sum of products of the form

$$
\left(\partial_{x}^{\alpha_{1}} \partial_{\xi}^{\alpha_{2}} p_{\xi}\right)\left(x_{t}, \xi_{t}\right) \prod_{k=1}^{\left|\alpha_{1}\right|}\left(\partial_{\xi}^{\alpha_{1}^{k}} x_{t}\right) \prod_{k=1}^{\left|\alpha_{2}\right|}\left(\partial_{\xi}^{\alpha_{2}^{k}} \xi_{t}\right), \quad\left(\partial_{x}^{\alpha_{1}} \partial_{\xi}^{\alpha_{2}} p_{x}\right)\left(x_{t}, \xi_{t}\right) \prod_{k=1}^{\left|\alpha_{1}\right|}\left(\partial_{\xi}^{\alpha_{1}^{k}} x_{t}\right) \prod_{k=1}^{\left|\alpha_{2}\right|}\left(\partial_{\xi}^{\alpha_{2}^{k}} \xi_{t}\right),
$$

where

$$
1 \leq\left|\alpha_{1}\right|+\left|\alpha_{2}\right| \leq|\alpha|, \quad 1 \leq\left|\alpha_{1}^{k}\right|,\left|\alpha_{2}^{k}\right| \leq|\alpha|-1, \quad \sum_{k}\left|\alpha_{1}^{k}\right|+\sum_{k}\left|\alpha_{2}^{k}\right|=|\alpha| .
$$

Then, given the induction hypothesis and the bounds (3.32), (3.31) we can bound $\bar{y}(t)$ and $\bar{\eta}(t)$ by

$$
\bar{y}(t) \leq c_{\alpha}(1+t \sqrt{\lambda})^{|\alpha|-1}, \quad\|\bar{\eta}\|_{L^{1}(0, t)} \leq c_{\alpha}(1+t \sqrt{\lambda})^{|\alpha|-1} .
$$

If we just use the boundedness of the linearized flow this gives a $(1+t \sqrt{\lambda})^{|\alpha|-1}$ bound for both $y$ and $\eta$. The additional $t$ factor for $y$ is obtained if we use this bound in the equation for $y$, which has bounded coefficients. 
We can use the above lemma to produce an expansion of $x_{t}, \xi_{t}$ in terms of powers of $t$ :

Lemma 3.6. Assume that the coefficients of $P$ satisfy $\nabla^{2} g \in L^{1}\left(L^{\infty}\right)$ with Fourier transform supported in $B(0, \sqrt{\lambda})$. Then the following estimates hold:

$$
\begin{gathered}
x_{t}=x+t p_{\xi}+t^{2} g(t, x, \xi), \\
\xi_{t}=\xi+t h(t, x, \xi),
\end{gathered}
$$

where $g, h$ satisfy the following bounds:

$$
\left|\partial_{\xi}^{\alpha} h(t, x, \xi)\right|,\left|\partial_{\xi}^{\alpha} g(t, x, \xi)\right| \leq(1+t \sqrt{\lambda})^{\alpha-1} .
$$

Proof. We have

$$
\begin{aligned}
x_{t} & =x+t p_{\xi}+\int_{0}^{t}(t-s) \frac{d^{2}}{d s^{2}} x_{s} d s \\
& =x+t p_{\xi}+\int_{0}^{t}(t-s) f\left(x_{s}, \xi_{s}\right) d s
\end{aligned}
$$

where $f$ can be computed from the flow equation,

$$
f(x, \xi)=p_{\xi x} p_{\xi}-p_{\xi \xi} p_{x} .
$$

It suffices to estimate the derivatives of the integrand in $L^{1}$,

$$
\left\|\partial_{\xi}^{\alpha} f\left(x_{t}, \xi_{t}\right)\right\|_{L^{1}(0, t)} \leq c_{\alpha} t(1+t \sqrt{\lambda})^{|\alpha|-1} .
$$

By (3.32), (3.31) we have

$$
\left\|\partial_{x}^{\alpha} \partial_{\xi}^{\beta} f(x, \xi)\right\|_{L^{1}\left(L^{\infty}\right)} \leq c_{\alpha, \beta}(\sqrt{\lambda})^{|\alpha|-1}
$$

so this can be easily done using the previous lemma. The estimate for $\xi_{t}$ is similar.

Another straightforward consequence of Lemma 3.5 is the following bound for the exponents in the kernel $G$.

Lemma 3.7. Let $G$ be as in (3.23). Then

$$
\left|\partial_{\xi}^{\alpha} G\left(x_{t}, \xi_{t}, y\right)\right| \leq c_{\alpha} e^{-c \lambda\left(\xi_{t}-y\right)^{2}}(1+t \sqrt{\lambda})^{|\alpha|} .
$$

In a similar manner we obtain the related result for the exponent in the oscillatory term in our kernel 4

Lemma 3.8. For $\xi$ in a compact set and away from 0 we have

$$
\left|\partial_{\xi}\left(\lambda\left(x_{t}-\tilde{y}\right) \xi_{t}\right)-\left(\lambda(x-\tilde{y})+t^{2} \lambda g(t, x, \xi)\right)\right| \leq\left(1+\lambda\left(x_{t}-\tilde{y}\right)^{2}\right)(1+t \sqrt{\lambda})
$$

and

$$
\left|\partial_{\xi}^{\alpha} \lambda\left(\left(x_{t}-\tilde{y}\right) \xi_{t}\right)\right| \leq c_{\alpha}\left(1+\lambda\left(x_{t}-\tilde{y}\right)^{2}\right)(1+t \sqrt{\lambda})^{|\alpha|}, \quad|\alpha| \geq 2 .
$$

\footnotetext{
${ }^{4}$ See [17] for an explicit computation.
} 


\section{Proof of Theorem 1.2}

By rescaling the problem reduces to the case when $T=1$ and

$$
\|g\|_{\mathcal{X}^{s}}^{2} \leq \mu^{2+s} .
$$

Now we follow the proof of Theorem 1.1 and successively simplify the problem by

(i) reduction to a dyadic estimate,

(ii) truncating the coefficients at frequency $\mu^{\frac{1}{2}} \lambda^{\frac{2}{2+s}}$.

However, when we reach this point we can directly use Theorem 1.1 to prove the result.

The Littlewood-Paley decomposition. The analysis below is related to the paradifferential calculus. Given a frequency $\lambda$ define the operator $P_{\lambda}$ as

$$
P_{\lambda}=\partial_{i} g_{\lambda}^{i j} \partial_{j}
$$

where $g_{\lambda}^{i j}$ are the coefficients of $P$ truncated at frequency $\frac{\lambda}{10}$,

$$
g_{\lambda}^{i j}=\left(1-U_{\frac{\lambda}{10}}\right) g^{i j}
$$

Our first step is to reduce the problem to the dyadic estimates

$$
\lambda^{-\rho-\frac{\sigma}{p}}\left\|S_{\lambda} v\right\|_{L^{p}\left(L^{q}\right)} \lesssim \mu^{\frac{1}{p}}\left\|S_{\lambda} v\right\|_{L^{\infty}\left(L^{2}\right)}+\lambda^{-1-\sigma} \mu^{-\frac{1}{p^{\prime}}}\left\|P_{\lambda} S_{\lambda} v\right\|_{L^{1}\left(L^{\infty}\right)} .
$$

This implies (1.9) provided we show that

i.e.

$$
\left\||D|^{-\sigma}\left(S_{\lambda} P-P_{\lambda} S_{\lambda}\right) u\right\|_{L^{1}\left(L^{2}\right)}^{2} \lesssim \mu\|\nabla u\|_{L^{\infty}\left(L^{2}\right)}^{2}
$$

$$
\left\||D|^{1-\sigma}\left(S_{\lambda} g-g_{\lambda} S_{\lambda}\right) \nabla u\right\|_{L^{1}\left(L^{2}\right)}^{2} \lesssim \mu\|\nabla u\|_{L^{\infty}\left(L^{2}\right)}^{2} .
$$

Since the range of $S_{\lambda} g-g_{\lambda} S_{\lambda}$ consists of functions frequency localized at frequency $\lambda$ this is equivalent to

$$
\left\|\left(S_{\lambda} g-g_{\lambda} S_{\lambda}\right) v\right\|_{L^{1}\left(L^{2}\right)} \lesssim \mu \lambda^{\sigma-1}\|v\|_{L^{\infty}\left(L^{2}\right)},
$$

where $v=\nabla u$. Because $v$ is compactly supported, without any restriction in generality we can assume that $g$ is compactly supported. Then we know that

$$
\|g\|_{L^{1}\left(L^{\infty}\right)} \leq 1, \quad\|g\|_{\mathcal{X}^{s}} \lesssim \mu^{\frac{2+s}{2}} .
$$

This implies the bound

$$
\|g\|_{\dot{B}_{1-\sigma}^{1 \infty 1}} \lesssim \mu
$$

where $1-\sigma=\frac{2 s}{2+s}$.

Hence (4.3) would follow from

$$
\left\|\left(S_{\lambda} g-g_{\lambda} S_{\lambda}\right) v\right\|_{L^{1}\left(L^{2}\right)} \leq \lambda^{\sigma-1}\|g\|_{\dot{B}_{1-\sigma}^{1 \infty 1}}\|v\|_{L^{\infty}\left(L^{2}\right)}
$$

It suffices to prove this for a single dyadic piece of $g$ at frequency $\nu$, which satisfies

$$
\left\|S_{\nu} g\right\|_{L^{1}\left(L^{\infty}\right)} \lesssim \nu^{-1+\sigma}\|g\|_{\dot{B}_{1-\sigma}^{1 \infty 1}}
$$

If $\nu \gg \frac{\lambda}{10}$, then we need to show that

$$
\left\|S_{\lambda}\left(S_{\nu} g\right) v\right\|_{L^{1}\left(L^{2}\right)} \lesssim \lambda^{\sigma-1} \nu^{1-\sigma}\left\|S_{\nu} g\right\|_{L^{1}\left(L^{\infty}\right)}\|v\|_{L^{\infty}\left(L^{2}\right)},
$$

which is straightforward.

If $\nu \lesssim \frac{\lambda}{10}$, on the other hand, then we need to show that

$$
\left\|\left[S_{\lambda},\left(S_{\nu} g\right)\right] v\right\|_{L^{1}\left(L^{2}\right)} \lesssim \lambda^{\sigma-1} \nu^{1-\sigma}\left\|S_{\nu} g\right\|_{L^{1}\left(L^{\infty}\right)}\|v\|_{L^{\infty}\left(L^{2}\right)} .
$$


But this is a scale invariant estimate. Without any restriction in generality we can take $\nu=1$ and show that

$$
\left\|\left[S_{\lambda},\left(S_{1} g\right)\right] v\right\|_{L^{1}\left(L^{2}\right)} \lesssim \lambda^{\sigma-1}\left\|S_{1} g\right\|_{L^{1}\left(L^{\infty}\right)}\|v\|_{L^{\infty}\left(L^{2}\right)} .
$$

We replace this by the stronger estimate

$$
\left\|\left[S_{\lambda}, g\right] v\right\|_{L^{1}\left(L^{2}\right)} \lesssim \lambda^{-1}\|\nabla g\|_{L^{1}\left(L^{\infty}\right)}\|v\|_{L^{\infty}\left(L^{2}\right)},
$$

which we rescale to the case $\lambda=1$ :

$$
\left\|\left[S_{1}, g\right] v\right\|_{L^{1}\left(L^{2}\right)} \lesssim\|\nabla g\|_{L^{1}\left(L^{\infty}\right)}\|v\|_{L^{\infty}\left(L^{2}\right)} .
$$

The last bound follows since the kernel $K(y, \tilde{y})$ of $\left[S_{1}, g\right]$,

$$
K(y, \tilde{y})=\hat{s}(y-\tilde{y})(g(y)-g(\tilde{y})),
$$

satisfies

$$
K(y, \tilde{y}) \lesssim\left(1+\left|y^{\prime}-\tilde{y}^{\prime}\right|\right)^{-N}\left(1+\left|y_{0}-\tilde{y}_{0}\right|\right)^{-N} \int_{y_{0}}^{\tilde{y}_{0}}\|\nabla g(s)\|_{\infty} d s
$$

and

$$
\int\left[\left(1+\left|y_{0}-\tilde{y}_{0}\right|\right)^{-N} \int_{y_{0}}^{\tilde{y}_{0}}\|\nabla g(s)\|_{\infty} d s\right] d y_{0} d \tilde{y}_{0} \lesssim\|\nabla g\|_{L^{1}\left(L^{\infty}\right)} .
$$

Truncating the coefficients. Now we show that it suffices to prove the dyadic estimate under the additional assumption that the coefficients of $P_{\lambda}$ have Fourier transform supported in $B\left(0, \mu^{\frac{1}{2}} \lambda^{\frac{2}{2+s}}\right)$. To achieve this we need to prove that if we truncate the coefficients at frequency $\mu^{\frac{1}{2}} \lambda^{\frac{2}{2+s}}$, then the change in the second right-hand side term in (4.2) is controlled by the first right-hand side term, i.e. that

$$
\left\|\partial\left(U_{\mu^{\frac{1}{2}} \lambda^{\frac{2}{2+s}}} g_{\lambda}\right) \partial S_{\lambda} u\right\|_{L^{1}\left(L^{2}\right)} \leq \mu \lambda^{1+\sigma}\left\|S_{\lambda} u\right\|_{L^{\infty}\left(L^{2}\right)} .
$$

Indeed,

$$
\begin{aligned}
\left\|\partial\left(U_{\mu^{\frac{1}{2}} \lambda^{\frac{2}{2+s}}} g_{\lambda}\right) \partial S_{\lambda} u\right\|_{L^{1}\left(L^{2}\right)} & \leq \lambda^{2}\left\|U_{\mu^{\frac{1}{2}} \lambda^{\frac{2}{2+s}}} g\right\|_{L^{1}\left(L^{\infty}\right)}\left\|S_{\lambda} u\right\|_{L^{\infty}\left(L^{2}\right)} \\
& \leq \lambda^{2}\left(\mu^{\frac{1}{2}} \lambda^{\frac{2}{2+s}}\right)^{-s}\|g\|_{\mathcal{X}^{s}}\left\|S_{\lambda} u\right\|_{L^{\infty}\left(L^{2}\right)} \\
& \leq \mu \lambda^{1+\sigma}\left\|S_{\lambda} u\right\|_{L^{\infty}\left(L^{2}\right)} .
\end{aligned}
$$

At the last step we have used the bound 4.1) for $g$.

Conclusion. Observe that if the coefficients of $P$ have Fourier transform supported in the region $\left\{|\xi| \leq \mu^{\frac{1}{2}} \lambda^{\frac{2}{2-s}}\right\}$, then

$$
\left\|\partial_{x}^{2} g\right\|_{L^{1}\left(L^{\infty}\right)} \leq \mu^{\frac{2-s}{2}} \lambda^{\frac{2(2-s)}{2+s}}\|g\|_{\mathcal{X}^{s}}
$$

which gives

$$
\left\|\partial_{x}^{2} g\right\|_{L^{1}\left(L^{\infty}\right)} \leq\left(\mu \lambda^{\sigma}\right)^{2} .
$$

Then (4.2) is a special case of (1.8). 


\section{QuAsilinear hyPERBolic EQUATIONS}

Consider a quasilinear second order hyperbolic equation in $\mathbb{R}^{n} \times \mathbb{R}$,

$$
\partial_{i} g^{i j}(u) \partial_{j} u=N(u, \nabla u)
$$

with Cauchy data

$$
u(0)=u_{0}, \quad u_{t}(0)=u_{1} .
$$

Then the classical theory (see [6], and also [19] and references therein) says that this problem is locally well-posed in $H^{s} \times H^{s-1}$ for $s>\frac{n}{2}+1$. This condition insures that the coefficients of the principal part are $C^{1}$ and that $\nabla u$ is bounded.

The Strichartz estimates in [17] allowed us to obtain the same result for a lower $s$ provided that the nonlinearity is at most quadratic in $\nabla u$,

$$
N(u, \nabla u)=G(u) Q(\nabla u, \nabla u) .
$$

The stronger estimates in this paper enable us to push this result even further. Results in the same direction were independently proved by H. Bahouri and J-Y. Chemin [2], 1] using a different method.

In what follows we assume that the functions $G, g^{i j}$ are smooth, bounded and have bounded derivatives up to a sufficiently high order. Also we assume that the coefficients $g^{i j}$ are uniformly hyperbolic in time. Then our main result is

Theorem 5.1. The quasilinear problem (5.1)-(5.2) is locally well-posed in $H^{s} \times$ $H^{s-1}$ for

$$
\begin{aligned}
& s>\frac{n}{2}+\frac{5}{6}, \quad n=2 \text {, } \\
& s>\frac{n}{2}+\frac{2}{3}, \quad n \geq 3 .
\end{aligned}
$$

One should note, though, that at this point the counterexamples for the quasilinear equation are no better than those for the semilinear equation; see [1], [12.

The same method can be used for second order hyperbolic equations of the form

$$
g^{i j}(u, \nabla u) \partial_{i} \partial_{j} u=N(u, \nabla u) .
$$

Differentiating once we obtain equations which are essentially of the form (5.1), therefore

Theorem 5.2. The quasilinear problem (5.4)-(5.2) is locally well-posed in $H^{s} \times$ $H^{s-1}$ for

$$
\begin{array}{ll}
s>\frac{n+2}{2}+\frac{5}{6}, & n=2, \\
s>\frac{n+2}{2}+\frac{2}{3}, & n \geq 3 .
\end{array}
$$

Proof of Theorem 5.1. The proof of this result is similar to the corresponding proof in [17, therefore here we only sketch the main arguments.

The energy estimates. A first step in the proof is to obtain good bounds for the $H^{s}$ norm of the solutions in terms of the initial data. The main estimate states that

$$
\|\nabla u(t)\|_{H^{s-1}} \leq c\|\nabla u(0)\|_{H^{s-1}} e^{c \int_{0}^{t}\|\nabla u(s)\|_{\infty} d s}
$$


This holds for any $s$ and implies that the solutions do not blow up in any $H^{s}$ norm for as long as

$$
\int_{0}^{t}\|\nabla u(s)\|_{\infty} d s
$$

remains finite, i.e. for as long as $\nabla u \in L^{1}\left(L^{\infty}\right)$. The proof of this result is also of interest to us. For $s=1$ it suffices to multiply the equation by $u_{t}$, integrate by parts and use Gronwall's lemma. For other values of $s$ we introduce the auxiliary function

$$
v=\left|D^{\prime}\right|^{s-1} u
$$

and compute

$$
P(x, u, D) v=\left[P(x, u, D)\left|D^{\prime}\right|^{s-1}\right] u+|D|^{s-1} \Gamma(u)(\nabla u)^{2} .
$$

A careful estimate of the right-hand side at fixed time yields

$$
\left\|\left[P(x, u, D)\left|D^{\prime}\right|^{s-1}\right] u+|D|^{s-1} \Gamma(u)(\nabla u)^{2}\right\|_{L^{2}} \leq\|\nabla u\|_{L^{\infty}}\|\nabla u\|_{H^{s-1}} .
$$

Then (5.5) follows from the $H^{1}$ energy estimates for $v$ combined with Gronwall's lemma.

This argument goes back to work of Klainerman [10] for integer $s$; for noninteger $s$ the proof of the estimate (5.6) requires better commutator estimates as proved later in 8 .

Thus, in order to obtain a local well-posedness result one has to determine for what $s$ is it possible to close the argument and recover locally in time the $L^{1}\left(L^{\infty}\right)$ regularity for $\nabla u$. This we do using the Strichartz estimates. In effect what we can get from the Strichartz estimates is $L^{4}\left(L^{\infty}\right)$ for $n=2, L^{2+\epsilon}\left(L^{\infty}\right)$ for $n=3$ respectively $L^{2}\left(L^{\infty}\right)$ for $n \geq 4$.

The Strichartz estimates. We apply the Strichartz estimates in Corollary 1.7 to the auxiliary function $v$ defined above. To fix the indices suppose for instance that $n \geq 4$. If we apriori assume that the coefficients satisfy

$$
\|\nabla g\|_{L^{1}\left(L^{\infty}\right)} \leq 1
$$

then we obtain locally

$$
\left\|\left|D^{\prime}\right|^{-\frac{n+1}{2}-\frac{1}{6}} \nabla v\right\|_{L^{2}\left(\dot{B}_{0}^{\infty \infty}\right)} \leq\|\nabla v(0)\|_{L^{2}}+\|P(x, u, D) v\|_{L^{1}\left(L^{2}\right)} .
$$

Combine this with the bound (5.6) for $P(x, u, D) v$ to get

$$
\left\|\left|D^{\prime}\right|^{-\frac{n+1}{2}-\frac{1}{6}} \nabla v\right\|_{L^{2}\left(\dot{B}_{0}^{\infty \infty}\right)} \leq c\left(1+\|\nabla u\|_{L^{1}\left(L^{\infty}\right)}\right)\|\nabla u\|_{L^{\infty}\left(H^{s-1}\right)},
$$

which for $u$ gives

$$
\|\nabla u\|_{L^{2}\left(L^{\infty}\right)} \leq c\left(1+\|\nabla u\|_{L^{1}\left(L^{\infty}\right)}\right)\|\nabla u\|_{L^{\infty}\left(H^{s-1}\right)}, \quad s>\frac{n}{2}+\frac{2}{3} .
$$

\footnotetext{
${ }^{5}$ Here we assume an apriori bound on the $L^{\infty}$ norm of $u$.
} 
Closing the loop. Since

$$
\|\nabla g\|_{L^{1}\left(0, T ; L^{\infty}\right)} \leq\|\nabla u\|_{L^{1}\left(0, T ; L^{\infty}\right)}
$$

all we need to do is to estimate $\nabla u$ in terms of itself and show that for a small time it has to stay in $L^{1}\left(L^{\infty}\right)$. We have

$$
\begin{aligned}
\|\nabla u\|_{L^{1}\left(0, T ; L^{\infty}\right)} & \leq \sqrt{T}\|\nabla u\|_{L^{2}\left(0, T ; L^{\infty}\right)} \\
& \leq \sqrt{T}\left(1+\|\nabla u\|_{L^{1}\left(0, T ; L^{\infty}\right)}\right)\|\nabla u\|_{L^{\infty}\left(0, T ; H^{s-1}\right)} \\
& \leq \sqrt{T}\left(1+\|\nabla u\|_{L^{1}\left(0, T ; L^{\infty}\right)}\right)\|\nabla u(0)\|_{H^{s-1}} e^{c \int_{0}^{T}\|\nabla u(t)\|_{L^{1}\left(0, T ; L^{\infty}\right)} d t} .
\end{aligned}
$$

Here we have used the Strichartz estimates on the second line, and the energy estimates on the third line.

Hence in order to recover our assumption that

$$
\|\nabla g\|_{L^{1}\left(0, T ; L^{\infty}\right)} \leq 1
$$

it suffices to choose $T$ so that

$$
\sqrt{T}\|\nabla u(0)\|_{H^{s-1}} \leq c \ll 1 .
$$

Thus for such a time $T$ we obtain uniform $H^{s}$ bounds on the solution if the initial data is in a bounded set in $H^{s}$ for $s>\frac{n}{2}+\frac{2}{3}$.

Stability estimates. To complete the proof of the well-posedness result we now show that the solutions depend continuously (even Lipschitz) on the initial data. Such a dependence cannot hold, though, in the $H^{s}$ norm. Here we contend ourselves with the energy norm.

Lemma 5.3. Assume that $n \geq 3$. Let $u, v$ be smooth solutions to (5.1) in $[0, T]$. Then

$$
\begin{aligned}
& \|\nabla(u-v)\|_{L^{\infty}\left(0, T ; L^{2}\right)} \\
& \quad \leq c\left(\|u\|_{H^{s}},\|v\|_{H^{s}},\|\nabla u\|_{L^{2}\left(L^{\infty}\right)},\|\nabla v\|_{L^{2}\left(L^{\infty}\right)}\right)\|\nabla(u-v)(0)\|_{L^{2}} .
\end{aligned}
$$

The proof is identical to the one in [17]. The difference

$$
w=u-v
$$

solves the linear equation

$$
P(x, u, D) w=A_{1} \nabla w+A_{0} w,
$$

where $A_{1}$ is linear in $\nabla u, \nabla v$,

$$
A_{1}=G_{1}(u, v) \nabla(u, v)
$$

while $A_{0}$ contains a term which is linear in the second order derivative of $v$ and a quadratic term in $\nabla u, \nabla v$,

$$
A_{0}=G_{2}(u, v) \partial^{\prime} \nabla v+G_{3}(u, v)(\nabla(u, v))^{2} .
$$

Using the Strichartz estimates for $u, v$ one can estimate the coefficients $A_{0}, A_{1}$ in mixed $L^{p}$ spaces. Then, due to the Strichartz estimates for $w$, it is easy to establish the $H^{1}$ well-posedness for (5.8) and further, to show that the right-hand side remains in $L^{2}$ locally. 
This argument is tight in dimension $n=3$. For $n \geq 4$ one has more freedom and the stability estimates are also valid in smaller Sobolev spaces. By contrast, for $n=2$ the above argument has to be modified since then $D_{x} \nabla v$ can only be estimated in negative Sobolev spaces. Thus, the best result one can obtain in a similar manner is a stability estimate in $H^{\frac{5}{6}} \times H^{-\frac{1}{6}}$.

\section{REFERENCES}

[1] Hajer Bahouri and Jean-Yves Chemin. Equations d'ondes quasilineaires et effet dispersif. Internat. Math. Res. Notices, 21:1141-1178, 1999. MR 2000i:35125

[2] Hajer Bahouri and Jean-Yves Chemin. Equations d'ondes quasilineaires et estimations de Strichartz. Amer. J. Math., 121(6):1337-1377, 1999. MR 2000i:35124

[3] Philip Brenner. On $L_{p}-L_{p^{\prime}}$ estimates for the wave-equation. Math. Z., 145(3):251-254, 1975. MR 52:8658

[4] Jean-Marc Delort. F.B.I. transformation. Second microlocalization and semilinear caustics. Springer-Verlag, Berlin, 1992. MR 93i:35010

[5] J. Ginibre and G. Velo. Generalized Strichartz inequalities for the wave equation. J. Funct. Anal., 133(1):50-68, 1995. MR 97a:46047

[6] Thomas J. R. Hughes, Tosio Kato, and Jerrold E. Marsden. Well-posed quasi-linear secondorder hyperbolic systems with applications to nonlinear elastodynamics and general relativity. Arch. Rational Mech. Anal., 63(3):273-294 (1977), 1976. MR 54:8041

[7] L.V. Kapitanskii. Estimates for norms in Besov and Lizorkin-Triebel spaces for solutions of second-order linear hyperbolic equations, Zap. Nauchn. Sem. Leningrad. Otdel. Mat. Inst. Steklov. (LOMI) 171:106-162, 185-186, 1989 MR 90m:35109

[8] Tosio Kato and Gustavo Ponce. Commutator estimates and the Euler and Navier-Stokes equations. Comm. Pure Appl. Math., 41(7):891-907, 1988. MR 90f:35162

[9] Markus Keel and Terence Tao. Endpoint Strichartz estimates. Amer. J. Math., 120(5):955980, 1998. MR 2000d:35018

[10] Sergiu Klainerman. Long-time behavior of solutions to nonlinear evolution equations. Arch. Rational Mech. Anal., 78(1):73-98, 1982. MR 84b:35015

[11] Hans Lindblad. Counterexamples to local existence for semi-linear wave equations. Amer. J. Math., 118(1):1-16, 1996. MR 97b:35124

[12] Hans Lindblad. Counterexamples to local existence for quasilinear wave equations. Math. Res. Lett., 5(5):605-622, 1998. MR 2000a:35171]

[13] Gerd Mockenhaupt, Andreas Seeger, and Christopher D. Sogge. Local smoothing of Fourier integral operators and Carleson-Sjölin estimates. J. Amer. Math. Soc., 6(1):65-130, 1993. MR 93h:58150

[14] Hart F. Smith. A parametrix construction for wave equations with $C^{1,1}$ coefficients. Ann. Inst. Fourier (Grenoble), 48(3):797-835, 1998. MR 99h:35119

[15] Hart F. Smith and Christopher D. Sogge. On Strichartz and eigenfunction estimates for low regularity metrics. Math. Res. Lett., 1(6):729-737, 1994. MR 95h:35156

[16] Robert S. Strichartz. Restrictions of Fourier transforms to quadratic surfaces and decay of solutions of wave equations. Duke Math. J., 44(3):705-714, 1977. MR 58:23577

[17] Daniel Tataru. Strichartz estimates for operators with nonsmooth coefficients and the nonlinear wave equation. Amer. J. Math., 122(2):349-376, 2000. MR 2001c:35260

[18] Daniel Tataru. Strichartz estimates for second order hyperbolic operators with nonsmooth coefficients II. Amer. J. Math., 123(3):385-423, 2001.

[19] Michael E. Taylor. Pseudodifferential operators and nonlinear PDE. Birkhäuser Boston Inc., Boston, MA, 1991. MR 92j:35193

Department of Mathematics, Northwestern University, Evanston, Illinois 60208 E-mail address: tataru@math.northwestern.edu

Current address: Department of Mathematics, University of California, Berkeley, California 94720

E-mail address: tataru@math.berkeley.edu 